is to be noted that the diffusion coefficients as calculated from viscosity data, of methyl and ethyl alcohols follow an Arrhenius temperature dependence with activation energies of about $4 \mathrm{kcal} / \mathrm{mole}$. While hydrogen bonding may well have a role in these liquids also, the absence of the extensive three-dimensional network probably makes its influence less important than in water.

\section{ACKNOWLEDGMENTS}

The authors wish to express thanks for helpful discussions with Dr. G. E. Walrafen and Dr. F. H. Stillinger, and technical assistance from Mr. D. R. Falcone.

* Present address: Department of Physics, University of the Witwatersrand, Jan-Smuts Ave., Johannesburg, South Africa.

${ }^{1}$ L. D. Eicher and B. J. Zwolinski, J. Phys. Chem. 75, 2016 (1971).

2 R. Hausser, G. Maier, and F. Noack, Z. Naturforsch. 21a, 1410 (1966).
${ }^{3}$ P. W. Bridgman, The Physics of High Pressure (G. Bell and Sons, London, 1958).

${ }^{4}$ A. A. Miller, J. Chem. Phys. 38, 1568 (1963).

${ }^{5}$ N. K. Gilra, Can. J. Phys. 46, 2123 (1968).

6 D. C. Douglass and D. W. McCall, J. Phys. Chem. 62, 1102 (1958).

${ }^{7}$ S. C. Mossop, Proc. Phys. Soc. (London) B68, 193 (1955).

${ }^{8}$ E. D. Stejskal and J. E. Tanner, J. Chem. Phys. 42, 288 (1965).

${ }_{9}^{9}$ R. Mills, Ber. Bunsenges. Physik. Chem. 75, 195 (1971) and references cited therein.

${ }^{10}$ D. E. O'Reilly and E. M. Peterson, J. Chem. Phys. 55, 2155 (1971).

11 N. J. Trappeniers, C. J. Gerritsma, and P. H. Oosting, Phys. Letters 18, 256 (1965).

${ }^{12}$ H. R. Pruppacher, J. Chem. Phys. 56, 101 (1972).

${ }^{13}$ N. H. Fletcher, The Chenical Physics of Ice (Cambridge U. P., New York, 1970), p. 160.

${ }_{14}$ G. E. Walrafen, in Water: A Comprehensive Treatise Vol. I, Physics and Physical Chemistry of Water, edited by Felix Franks (Plenum, New York, 1972).

${ }^{15}$ W. A. P. Luck and W. Ditter, J. Phys. Chem. 74, 3687 (1970).

${ }^{16}$ G. Houghton, J. Chem. Phys. 40, 1628 (1964).

$17 \mathrm{~J}$. W. Hennel and K. Krynicki, Acta Phys. Polon. 18, 523 (1959).

THE JOURNAL OF CHEMICAL PHYSICS

VOL UME 57, N U M B E R 12

15 DE C E M B E R 1972

\title{
Strengths and Collision Broadened Widths in the Second Overtone Band of Hydrogen Fluoride*
}

\author{
R. L. Spelicicy, R. E. Meredith, $\dagger$ and F. G. Sartit $\dagger$
}

Willow Run Laboralories, Institute of Science and Technology, The University of Michigan, P.O. Box 618, Ann Arbor, Michigan 48107

(Received 6 June 1972)

\begin{abstract}
Individual line strengths and self-broadened half-widths have been measured in the second overtone band of hydrogen fluoride. The electric dipole matrix element for the band has been determined from the measured strengths. Its value is:
\end{abstract}

$$
\langle 3|\mu(r)| 0\rangle_{\text {exD }}=+1.628 \times 10^{-21} \mathrm{esu} \cdot \mathrm{cm} \text {. }
$$

The $m$ dependence of the measured half-widths agree with the Anderson theory of collision broadening if off resonant collisions are taken into account.

\section{INTRODUCTION}

Collision broadened molecular vibration-rotation line parameters continue to be of importance for a variety of applications. For example, strength and width parameters are required for analytical predictions of radiative transfer within complex gaseous mixtures. Also, the electric dipole matrix element of a molecular transition may be determined from line strengths measured by absorption spectroscopy, and the electric dipole moment function may be empirically modeled using the matrix elements. ${ }^{1-3}$ Determination of the dipole moment permits the calculation of radiative lifetimes and absorption strengths of lines not measurable in the laboratory, e.g., fundamental and overtone vibration-rotation lines involving levels for which $v>0$, and for which $J$ is not sufficiently populated to permit observation. Collision broadened line halfwidths supply information about molecular collision processes particularly with regard to the validity of collision broadening and collision deactivation theories. Also, measured line half-widths may yield molecular electric multipole parameters of the active species and its collision partners.

In the present investigation, line strengths, and selfbroadened half-widths in the second overtone band of hydrogen fluoride have been measured. The primary motivation of the investigation was to obtain a better definition of the $\mathrm{HF}$ dipole moment ${ }^{4}$ for the calculation of radiative lifetimes of vibration-rotation lines lying well above the ground state. These lifetimes are required for an understanding of $\mathrm{HF}$ chemical laser performance as well as other applications for which radiative strengths of excited $\mathrm{HF}$ are required.

\section{MATRIX ELEMENT CALCULATIONS}

The connection between the strength of a vibrationrotation spectral absorption line and its electric dipole 
matrix is, for a diatomic molecule,

$$
S_{v_{u}, v l}(m)=\frac{8 \pi^{3}|m| \nu \exp \left[-T\left(v_{l}, J_{l}\right) h c / k T\right]\left|\left\langle v_{u} J_{u}|\mu(r)| v_{l} J_{l}\right\rangle\right|^{2}}{3 h c k T Z} \times 1.0135 \times 10^{6}
$$

The matrix element $\left|\left\langle v_{u} J_{u}|\mu(r)| v_{l} J_{l}\right\rangle\right|$ is given by the expression

$$
\left|\left\langle v_{u} J_{u}|\mu(r)| v_{l} J_{l}\right\rangle\right|=\int \psi_{v_{u} J_{u}} \mu(r) \psi_{v_{l} J} \boldsymbol{r}^{2} d r .
$$

The symbols used in the expressions above are as follows: $S$ is the line strength in units $\left(\mathrm{atm} \cdot \mathrm{cm}^{2}\right)^{-1}$, $\nu$ is the wavenumber of the transition, $Z$ is the vibration-rotation partition function, $T\left(\nu_{l}, J_{l}\right)$ is the term value of lower level, $k$ is Boltzmann's constant, $T$ is the temperature in deg. Kelvin, $\mu(r)$ is the radial electric dipole moment function, and $m$ is $-J_{l}$ for the $P$ branch, $+J_{u}$ for the $R$ branch.

The matrix elements are functions of the internuclear potential through the wavefunction $\psi(r)$, and they are functions of the form of the electric dipole moment $\mu(r)$. The most commonly used function $\mu(r)$ is the truncated polynomial expansion $\mu(n p)$

$$
\mu(n p)=\sum_{n} M_{n}\left(r-r_{\varepsilon}\right)^{n} .
$$

The matrix elements $\pm\left\langle v_{u} J_{u}|\mu(r)| v_{l} J_{l}\right\rangle_{\exp }$ may be determined from individual line strengths $S(m)$ from Eq. (1). The line strengths may be obtained from the measured absorption coefficient of the transition, $k(\nu)$, through the relationship

$$
S(m)=P^{-1} \int_{\text {line }} k(\nu) d \nu .
$$

Where $P$ is the pressure of the absorbing gas. On the other hand, the matrix elements (and hence the line strengths) may be calculated if the polynomial coefficients $M_{n}$, and the wavefunctions $\psi$ are known. ${ }^{4}$

In the present investigation, the parameters $M_{n}$ have been determined from line strength measurements in various overtone bands through simultaneous solution of equations of the form

$$
\begin{aligned}
& \langle v|\mu(r)| 0\rangle=\sum_{i=0}^{v_{\max }} M_{i} \int \psi_{v}\left(r-r_{s}\right)^{i} \psi_{0} r^{2} d r, \\
& \text { for } v=0,1 \cdots v_{\max } .
\end{aligned}
$$

Where $v_{\max }$ is the upper vibrational quantum number of the highest overtone measurement available. The matrix elements have been calculated by numerical integration of Eq. (2). Wavefunctions $\psi_{b, J}$ were obtained by solving Schrödinger's equation using finite difference equations. The RKR potential function was used including the vibration-rotation interaction term $J(J+1) / r^{2}$.

\section{LINEWIDTH CALCULATIONS}

The Anderson theory has been used with considerable success to calculate self-broadened half-widths of spectral lines of simple molecules., ${ }^{3,5,6}$ In this theory, the half-width $(\gamma)$ is related to the real part of the total cross section $(\sigma)$ through the relation

$$
\gamma(m)=(n \bar{v} / 2 c)(\sigma / \pi)
$$

where $\bar{v}$ is the mean relative collision velocity and $n$ is the number density at one atmosphere and at temperature $T$. The cross section is written as the statistical average of the partial cross sections of each rotational state $\left(J_{2}\right)$ of the perturbing molecule.

$$
\sigma / \pi=Z^{-1} \sum_{J_{2}}\left[\sigma\left(J_{2}\right) / \pi\right] g \exp \left(-E_{J_{2}} h c / k T\right),
$$

where $g$ is the degeneracy of the perturbing state. The partial cross sections are in turn related to the impact parameters $b\left(J_{2}\right)$ and $S(b)$ which contains the details of the collision through the relationship

$$
\frac{\sigma\left(J_{2}\right)}{\pi}=2 \int_{0}^{\infty} S(b) b d b .
$$

In what follows, the functional dependence of $b\left(J_{2}\right)$ on $J_{2}$ will be suppressed in the interest of clarity.

$S(b)$ may be expressed in terms of multipole interactions for $b>0$ as

$$
S(b)=\sum_{J_{u} J_{l}}\left[\sum_{s=1}^{3} \sum_{t} C_{s} b^{-2(s+1)} g_{s t} f_{s}\left(k_{s t}\right)\right],
$$

$s=1,2$, and 3 represent the dipole-dipole, dipolequadrupole, and quadrupole-quadrupole contributions, respectively. The $g_{s t}$ are functions of Clebsch-Gordan coefficients, and the $f_{8}(k)$ are resonance factors. ${ }^{6.7}$ The parameter $k$ is essentially the energy "defect" of a near resonant collision. The coefficients $C_{s}$ and the parameter $k$ are as follows,

$$
\begin{aligned}
C_{1} & =(4 / 9)\left[\mu_{0}^{4} /(\hbar \bar{v})^{2}\right], \\
C_{2} & =(4 / 45)\left[\mu_{0}^{2} Q^{2} /(\hbar \bar{v})^{2}\right], \\
C_{3} & =(1 / 25)\left[Q^{4} /(\hbar \bar{v})^{2}\right], \\
k_{s, l} & =(2 \pi c b / \bar{v})\left|(\Delta E)_{s t}\right| .
\end{aligned}
$$

For polar molecules such as HF, Anderson's approximation \# $2^{5}$ is appropriate

$$
\begin{array}{cc}
S(b)=1 & b \leq b_{0}, \\
S(b)<1 & b>b_{0} .
\end{array}
$$

Integration of Eq. (8) then gives

$$
\sigma\left(J_{2}\right) / \pi=b_{0}^{2}+\sum_{t, s} C_{s} b_{0}^{-2 s} g_{s t} F_{s}\left(k_{0}\right),
$$

where $k_{0}$ is the $k_{s t}$ evaluated at $b\left(J_{2}\right)=b_{0}\left(J_{2}\right)$, and where Eq. (11) has been applied to Eq. (9) to determine $b_{0}\left(J_{2}\right)$. 
In practice, Eq. (12) can be used only for collision partners $J_{2}$ for which the long range forces dominate. If this is not the case, $b_{0}$ must be determined empirically, or a model such as the resonant dipole billiard ball model must be used. ${ }^{8}$

TABLE I. Measured line parameters.

\begin{tabular}{|c|c|c|c|}
\hline \multirow[b]{2}{*}{ Line } & \multirow[b]{2}{*}{ Parameter } & \multicolumn{2}{|c|}{ Pressure } \\
\hline & & $0.467 \mathrm{~atm}$ & $0.932 \mathrm{~atm}$ \\
\hline$P(4)$ & $\begin{array}{l}S \\
\gamma / P \\
k^{P}\end{array}$ & $\begin{array}{l}\cdots \\
\cdots \\
\cdots\end{array}$ & $\begin{array}{l}0.0175 \mathrm{~cm}^{-2} \cdot \mathrm{atm}^{-1} \\
0.430 \mathrm{~cm}^{-1} \cdot \mathrm{atm}^{-1} \\
0.0127 \mathrm{~cm}^{-1}\end{array}$ \\
\hline$P(3)$ & $\begin{array}{l}S \\
\gamma / P \\
k^{P}\end{array}$ & $\begin{array}{l}0.0235 \\
0.503 \\
0.0149\end{array}$ & $\begin{array}{l}0.0232 \\
0.502 \\
0.0144\end{array}$ \\
\hline$P(2)$ & $\begin{array}{l}S \\
\gamma / P \\
k^{P}\end{array}$ & $\begin{array}{l}0.0245 \\
0.506 \\
0.0154\end{array}$ & $\begin{array}{l}0.0235 \\
0.509 \\
0.0146\end{array}$ \\
\hline$P(1)$ & $\begin{array}{l}S \\
\gamma / P \\
k^{P}\end{array}$ & $\begin{array}{l}0.0164 \\
0.447 \\
0.0116\end{array}$ & $\begin{array}{l}0.0164 \\
0.488 \\
0.0107\end{array}$ \\
\hline$R(0)$ & $\begin{array}{l}S \\
\gamma / P \\
k^{P}\end{array}$ & $\begin{array}{l}0.0193 \\
0.397 \\
0.0154\end{array}$ & $\begin{array}{l}0.0188 \\
0.430 \\
0.0139\end{array}$ \\
\hline$R(1)$ & $\begin{array}{l}S \\
\gamma / P \\
k^{P}\end{array}$ & $\begin{array}{l}0.0327 \\
0.443 \\
0.0234\end{array}$ & $\begin{array}{l}0.0310 \\
0.427 \\
0.0231\end{array}$ \\
\hline$R(2)$ & $\begin{array}{l}S \\
\gamma / P \\
k^{P}\end{array}$ & $\begin{array}{l}0.0353 \\
0.453 \\
0.0248\end{array}$ & $\begin{array}{l}0.0308 \\
0.400 \\
0.0245\end{array}$ \\
\hline$R(3)$ & $\begin{array}{l}S \\
\gamma / P \\
k^{P}\end{array}$ & $\begin{array}{l}0.0287 \\
0.412 \\
0.0222\end{array}$ & $\begin{array}{l}0.0299 \\
0.434 \\
0.0219\end{array}$ \\
\hline$R(4)$ & $\begin{array}{l}S \\
\gamma / P \\
k^{P}\end{array}$ & $\begin{array}{l}0.0188 \\
0.331 \\
0.0182\end{array}$ & $\begin{array}{l}0.0159 \\
0.295 \\
0.0172\end{array}$ \\
\hline$R(5)$ & $\begin{array}{l}S \\
\gamma / P \\
k^{P}\end{array}$ & $\begin{array}{l}0.0099 \\
0.254 \\
0.0124\end{array}$ & $\begin{array}{l}0.0103 \\
0.256 \\
0.0127\end{array}$ \\
\hline$R(6)$ & $\begin{array}{l}S \\
\gamma / P \\
k^{P}\end{array}$ & $\begin{array}{l}0.0039 \\
0.164 \\
0.0074\end{array}$ & $\begin{array}{l}0.0038 \\
0.167 \\
0.0072\end{array}$ \\
\hline
\end{tabular}

\section{EXPERIMENTAL}

Measurements were performed with a three meter focal length EBERT spectrometer constructed by Marshall.9 A 600 groove $/ \mathrm{mm}$ Bausch and Lomb replica grating blazed at $1.6 \mu \mathrm{m}$ was used in second order, double passed. An average spectral resolution of $0.07 \mathrm{~cm}^{-1}$ across the second overtone band of $\mathrm{HF}$ was obtained.
TABLE II. Experimental matrix elements.

\begin{tabular}{ll}
\hline & $\left\langle v^{\prime} J^{\prime}|\mu(\boldsymbol{r})| \nu J\right\rangle_{\text {oxp }}$ \\
\hline$P(4)$ & $1.738 \times 10^{-21} \mathrm{esu} \cdot \mathrm{cm}$ \\
$P(3)$ & 1.677 \\
$P(2)$ & 1.642 \\
$P(1)$ & 1.639 \\
$R(0)$ & 1.623 \\
$R(1)$ & 1.613 \\
$R(2)$ & 1.563 \\
$R(3)$ & 1.615 \\
$R(4)$ & 1.528 \\
$R(5)$ & 1.574 \\
$R(6)$ & 1.554 \\
\hline
\end{tabular}

Wavelength calibration was achieved using the HF lines themselves since accurate line positions are well known. ${ }^{10,11}$ Resolution checks were made using krypton and xenon Geissler tube emission lines.

Determination of scattered light is particularly difficult in this spectral region $\left(11500 \mathrm{~cm}^{-1}\right)$ since molecular absorption strengths are too weak to permit observation of opaque lines, without very long path lengths. It was found that the required extinction could be obtained from a 0.09 molar concentration of neodymium $(+3)$ in a 1 normal solution of nitric acid. Although the absorption lines of $\mathrm{Nd}^{+3}$ are broad, it was found that the strongest of the lines could be made sufficiently opaque while leaving sufficient window transmittance. Scattered light determined in this way was less than $1 \%$.

A silicon photo-voltaic detector, cooled to $-79^{\circ} \mathrm{C}$, was used for all measurements. Light from a $1000 \mathrm{~W}$ quartz iodine source was modulated at $90 \mathrm{~Hz}$, and the signal was amplified by a Princeton Applied Research

TABLE III. Dipole moment parameters and experimental matrix elements.

\begin{tabular}{|c|c|c|c|}
\hline & \multicolumn{3}{|c|}{$\left(r_{o}=0.91717 \AA\right)$} \\
\hline & $\mu(1 p)$ & $\mu(2 p)$ & $\mu(3 p)$ \\
\hline$M_{0}$ & 1.7957 & 1.7966 & 1.7965 \\
\hline$M_{1}$ & 1.4968 & 1.5093 & 1.5229 \\
\hline$M_{2}$ & $\cdots$ & -0.2372 & -0.0806 \\
\hline$M_{3}$ & $\cdots$ & $\cdots$ & -1.4077 \\
\hline \multicolumn{4}{|c|}{$M_{i}=\left(10^{8} / \mathrm{cm}\right)^{i} \mathrm{D}$} \\
\hline \multicolumn{4}{|c|}{$\langle 0|\mu(r)| 0\rangle_{\text {exp }}=1.819 \times 10^{-18} \mathrm{esu} \cdot \mathrm{cm}$} \\
\hline \multicolumn{4}{|c|}{$\langle 1|\mu(r)| 0\rangle_{\text {oxp }}=9.850 \times 10^{-20} \mathrm{esu} \cdot \mathrm{cm}^{\mathrm{a}}$} \\
\hline \multicolumn{4}{|c|}{$\langle 2|\mu(r)| 0\rangle_{\text {oxp }}=-1.253 \times 10^{-20} \mathrm{esu} \cdot \mathrm{cm}^{\mathrm{b}}$} \\
\hline \multicolumn{4}{|c|}{$\langle 3|\mu(r)| 0\rangle_{\text {oxp }}=+1.628 \times 10^{-21} \mathrm{esu} \cdot \mathrm{cm}$} \\
\hline
\end{tabular}

s Reference 15.

b Reference 3. 


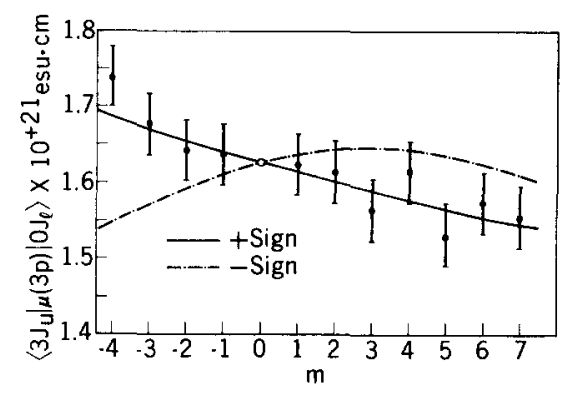

Fig. 1. Comparison of measured and calculated hydrogen fluoride matrix elements.

Corporation lock in amplifier, Mod. HR8, and was displayed on a Leeds and Northrup chart recorder.

An absorption cell having an optical path length of $33 \mathrm{~cm}$ was used for all measurements. The cell was constructed of monel with sapphire windows and had a design identical to that reported earlier. ${ }^{3}$ The gas handling system was also similar to that described in Ref. 3. As before, the cell, gas manifold, and a teledyne pressure transducer were maintained at $100^{\circ} \mathrm{C}$ while the cell was filled with $\mathrm{HF}$, and, during the measurements, the cell was immersed in a bath of gently boiling water. These and other gas handling precautions were used to avoid the effects of polymerization of $\mathrm{HF}$ at room temperature, the absorption of HF by the cell walls, and the reaction of $\mathrm{HF}$ with exposed metal surfaces.

Observed linewidths, peak values of absorption coefficient, and integrated absorption coefficients were taken directly from the chart recorder and corrected by the direct measurement method. The entire procedure was the same as described in an earlier publication. ${ }^{12}$

\section{EXPERIMENTAL STRENGTHS, WIDTHS, AND MATRIX ELEMENTS}

Line strengths, widths, and peak absorption coefficients were determined by direct measurement using

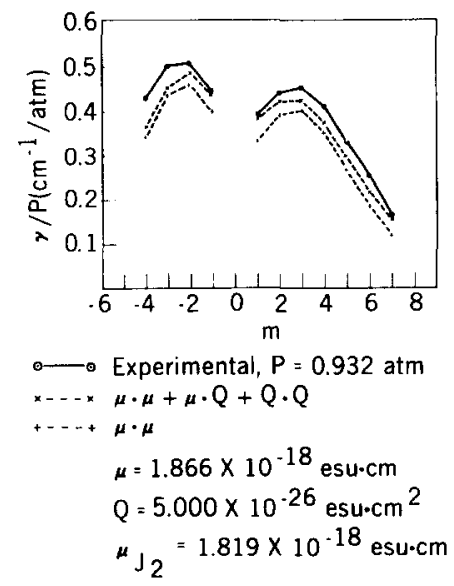

FIG. 2. Comparison of measured and calculated half-widths. correction factors tabulated in Ref. 12. Table I shows the corrected values for pressures of 0.932 and 0.467 atm. Dipole moment matrix elements, given in Table II, were obtained by averaging the measured strengths and using Eq. (1).

Since band strength measurements determine only the square of the rotationless matrix elements, the $m$ dependence of the matrix elements must be used to remove the sign ambiguity. This is feasible in the case of $\mathrm{HF}$ since its vibration-rotation interaction is quite large. The signs determined in this manner are listed in Table III along with the present value of $\langle 3|\mu(r)| 0\rangle_{\exp }$ and the preferred dipole moment coefficients of $\mu(3 p)$. The correctness of this choice of sign is indicated in Fig. 1 where the experimental matrix elements are compared with calculations using both the preferred $\mu(3 p)$ and that based on the negative matrix element. The wavefunctions used in the calculations were computed numerically using the RKR potential tabulated in Ref. 4. This potential was

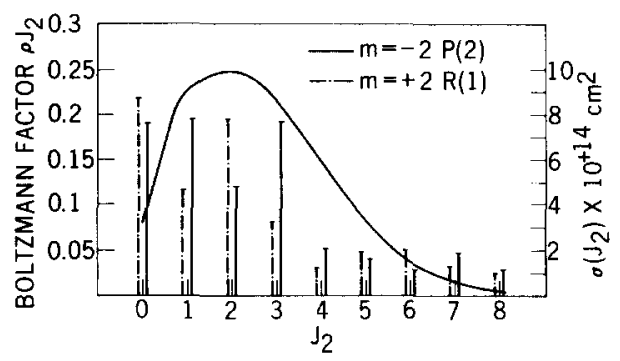

FIG. 3. Origin of the difference between $P$ and $R$ branch halfwidths.

calculated with a program written by Zare ${ }^{13}$ using the spectroscopic data of Johns and Barrow ${ }^{14}$ as input.

A comparison of the observed and calculated halfwidths is shown in Fig. 2. Listed also are the dipole and quadrupole moments used in the calculations. For the radiating molecule, $\mu$ was taken as a weighted average between its $v=0$ and $v=3$ values while the $v=0$ dipole moment was used for the perturbing molecule. The same quadrupole moment was used in both cases. The lower curve shows the widths obtained from Eq. (6) considering only dipole-dipole contributions. The upper curve includes contributions through quadrupole-quadrupole. The comparison is quite good, indicating that the theory properly accounts for the energy defects. As predicted by the theory and verified with the fundamental band and first overtone band of $\mathrm{HF}^{3}$, the $R$ branch lines are narrower than the corresponding $P$ branch lines, for small $|m|$, and the linewidths decrease progressively with increasing $\Delta v$. The tendency of the calculated values to lie below the experimental curve is not unexpected, since forces of shorter range than quadrupole have not been explicitly accounted for. 
The $m$ dependence of the linewidths arises from the product of the $J_{2}$ dependent collision cross section and the Boltzmann factor. This is shown in Figs. 3 and 4 . Figure 3 depicts the case where the spectral line involves energy levels near the Boltzmann maximum. The Boltzmann distribution for the $v=0$ levels of the colliding molecule and the partial cross sections contributed by the $J_{2}$ states are shown. It is seen that the contributions for $m=+2$ and $m=-2$ are not identical and that the Boltzmann distribution is more favorable for the $P$ branch lines, causing it to be broader than the corresponding $R$ branch line.

Figure 4 describes the case of lines whose energy levels are far from the Boltzmann maximum. The partial cross sections for the two branches are more comparable in this case, and the Boltzmann distribution does not favor either branch. It can be seen that the resonant and near resonant terms are eliminated by the Boltzmann factor and that the dominant contributions are now the off resonant terms. These terms are nearly equal and tend to a constant value as the energy defect $\Delta E$ increases. This produces nearly equal half-widths for the $P$ and $R$ branches for large $|m|$. This is essentially the assumption of the resonant dipole billiard ball model (RDBBM) ${ }^{8}$ and is in agreement with measured first overtone half-widths. If it is assumed that the constant value of the cross section is given by $\sigma\left(J_{2}\right)=\pi b_{\mathrm{Min}^{2}}{ }^{2}$, the calculations predict a value $b_{\mathrm{M} \text { in }} \simeq 5.6 \times 10^{-8} \mathrm{~cm}$. This compares well with the half-widths reported in Ref. 3.

The contributions of the higher order terms (dipolequadrupole, quadrupole-quadrupole) may be inferred from Fig. 2. Although these terms are not as large as the resonant or near-resonant dipole terms, they become increasingly significant as the stronger terms move into the wings of the Boltzmann distribution. Figure 5 clarifies this by showing the partial cross sections calculated from dipole-dipole interactions alone and those calculated using all interactions through quadrupole quadrupole. The increase in $\sigma(1), \sigma(2), \sigma(3)$, and $\sigma(4)$ due to the higher order terms, significantly alters the calculated half-widths

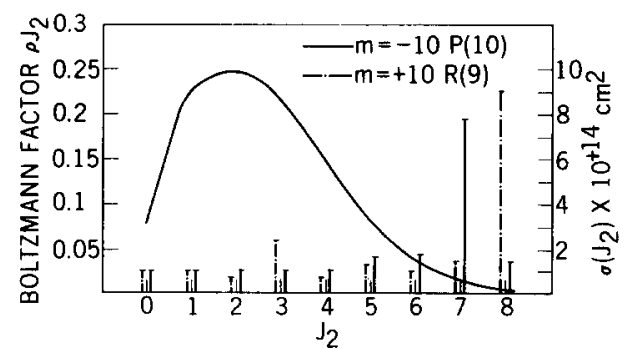

FIG. 4. Explanation of the "billiard ball" parameter $b_{\min }$.

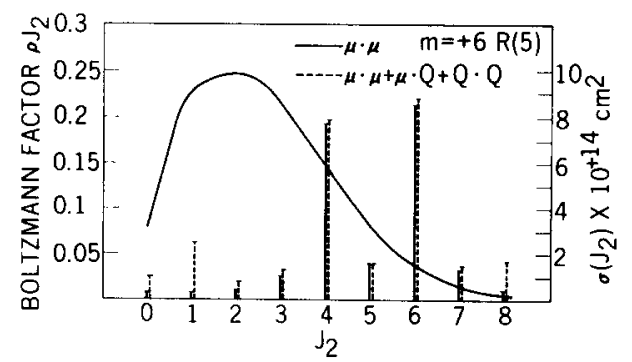

FIG. 5. Influence of the $\mu \cdot Q$ and $Q \cdot Q$ interactions on the halfsince these levels are heavily weighted by the Boltzmann factor.

\section{CONCLUSIONS}

This investigation confirms earlier indications that self-broadened spectral linewidths can be reliably predicted by Anderson-type theories using long-range multipolar molecular interactions. Although the $\mathbf{H F}$ molecule represents the extreme case of small moment of inertia and large dipole moment, the striking agreement between theory and experiment indicates that linewidths may be calculated accurately if proper account is taken of nonresonant effects. It is also confirmed that the effects of vibration-rotation interaction diminish with increasing $\Delta v$, in agreement with theory.

\section{ACKNOWLEDGMENTS}

The authors wish to acknowledge the cooperation of The University of Michigan Physics Department. Particular thanks is due Professor C. W. Peters for his interest and helpful discussion.

* This research was sponsored by the Advanced Research Projects Agency under Contract DAAH01-72-C-0573, ARPA Order 1108 .

† Present address: Science Applications Incorporated, 5 Research Drive, P. O. Box 328, Ann Arbor, Michigan 48103.

1 W. S. Benedict, J. Phys. 34, 830 (1956).

2 R. Herman and R. F. Wallis, J. Chem. Phys. 23, 637 (1955).

${ }^{3}$ R. E. Meredith, J. Quant. Spectry. Radiative Transfer 12, 485 (1972).

${ }^{4}$ R. E. Meredith and F. F. Smith, J. Quant. Spectry. Radiative Transfer (to be published).

5 P. W. Anderson, Phys. Rev. 76, 647 (1949).

${ }^{6}$ W. S. Benedict and R. Herman, J. Quant. Spectry. Radiative Transfer 3, 265 (1963)

${ }^{7}$ C. J. Tsao and B. Curnutte, J. Quant. Spectry. Radiative Transfer 2, 41 (1962).

${ }_{8}$ W. S. Benedict, et al., Can. J. Phys. 34, 850 (1956).

H. W. Marshall, Ph.D. dissertation, 1960, University of Michigan (unpublished).

${ }_{10}$ W. F. Herget, et al., J. Opt. Soc. Am. 52, 1113 (1962).

11 E. S. Fishburne and K. Narahari Rao, J. Mol. Spectry. 19, 290 (1966).

${ }_{12}$ R. E. Meredith, J. Quant. Spectry. Radiative Transfer 12, 455 (1972).

${ }_{13}$ R. N. Zare, UCRL Rept. No. UCRL-10925, November 1963.

${ }_{14}^{14}$ J. W. C. Jones and R. F. Barrow, Proc. Roy. Soc. (London) A251, 504 (1959). 